\title{
Synthesis and Antifungal Activity of New Bis- $\gamma$-lactones Analogous to Avenaciolide
}

\author{
Andréia da Silva Magaton, ${ }^{a}$ Mayura M. M. Rubinger, ${ }^{*},{ }^{b}$ Fernando C. de Macedo Júnior ${ }^{a}$ and \\ Laércio Zambolim ${ }^{b}$ \\ ${ }^{a}$ Departamento de Química and ${ }^{b}$ Departamento de Fitopatologia, Universidade Federal de Viçosa, \\ 36570-000 Viçosa-MG, Brazil
}

\begin{abstract}
Com o objetivo de avaliar o potencial agroquímico de uma série de compostos com atividade fungicida, foram preparadas e caracterizadas por análises elementares e espectroscopias no infravermelho e de RMN, três novas bis- $\gamma$-lactonas análogas ao avenaciolídeo, onde o grupo octila presente nesse produto natural foi substituído por grupos heptila, hexila e pentila. Os efeitos desses compostos e de seus precursores sintéticos no desenvolvimento micelial e na germinação de conídias de Colletotrichum gloesporioides foram avaliados in vitro. As bis- $\gamma$ lactonas análogas ao avenaciolídeo foram ativas, enquanto todos os precursores sintéticos não apresentaram atividade nas condições testadas. Este artigo descreve, ainda, a preparação e caracterização de 15 intermediários sintéticos inéditos.
\end{abstract}

In a study of the antifungal activity of selected compounds as potentials agrochemicals, we have prepared and characterized by elemental analyses, infrared and NMR spectroscopies three new bis- $\gamma$-lactones analogous to avenaciolide, where the octyl group of this natural product was replaced by heptyl, hexyl and pentyl groups. The effects on the mycelia development and conidia germination of Colletotrichum gloesporioides of these compounds and their synthetic precursors were evaluated in vitro. The title compounds were active in the tested conditions, while all the synthetic precursors were inactive. The preparation and characterization of 15 new synthetic intermediates are also described.

Keywords: bis- $\gamma$-lactones, antifungal activity, Colletotrichum gloesporioides

\section{Introduction}

Avenaciolide (Figure 1) is a natural product isolated from Aspergillus avenaceus, which possesses several interesting biological activities such as antifungal, antibacterial activities and inhibition of the glutamate transport in mitochondria. ${ }^{1-7}$ Isoavenaciolide, a related natural bis- $\gamma$-lactone which differs from avenaciolide by the orientation of the side chain (Figure 1), is about five times less effective than avenaciolide as an inhibitor of the glutamate transport while the analogue ethisolide is inactive. ${ }^{2,8}$ These findings suggest the crucial role played by the configuration of the C-6 stereogenic center and the length of the hydrophobic alkyl side chain on the biological activities.

A review on the synthesis of avenaciolide and related metabolites has been published by Martin et al. ${ }^{9}$ Probably due to all the interesting biological

*e-mail: mayura@ufv.br activities presented by avenaciolide, different methodologies are still being proposed for the synthesis of this class of compounds. ${ }^{10}$ Using the synthetic approach described by Anderson and Fraser-Reid for the preparation of the natural product from diacetoneD-glucose, ${ }^{11}$ we have prepared three avenaciolide analogues (Compounds 7, Scheme 1) which present side chains of different lengths. These compounds and the new synthetic intermediates (Compounds 3 to 6 , Scheme 1) were characterized by elemental analyses, infrared and NMR spectroscopies. The effects of compounds 3 to 7 on the germination of conidia and on the mycelia development of Colletotrichum gloeosporioides were evaluated in vitro.

\section{Results and Discussion}

The synthetic approach shown in Scheme 1 was used in the preparation of compounds $7(\mathbf{a}, \mathbf{b}, \mathbf{c})$, which present linear side chains of five, six and seven carbon 


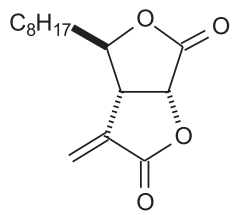

Avenaciolide

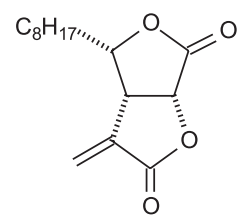

Isoavenaciolide

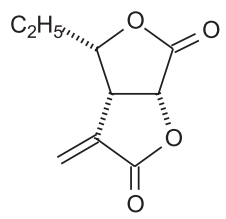

Ethisolide
Figure 1. Three natural fungi metabolites presenting bis- $\gamma$-lactone skeletons.

atoms, respectively. The aldehyde intermediate 2 was prepared from diacetone-D-glucose $\mathbf{1}$ as described in the literature. ${ }^{12,13}$

The alkyl side chains were introduced by the Wittig reaction between the aldehyde $\mathbf{2}$ and the ylides prepared in situ from alkyltriphenylphosphonium bromide and butyl lithium, yielding the compounds $\mathbf{3}(\mathbf{a}, \mathbf{b}, \mathbf{c})$. The signals due to the olefinic carbons 1" and 2" (Scheme 1) were observed at $c a . \delta 137$ and 126 , respectively, in the ${ }^{13} \mathrm{C}$ NMR spectra of compounds $\mathbf{3}$ (a, b, c). The coupling constant of $10.9 \mathrm{~Hz}$ observed in the ${ }^{1} \mathrm{H}$ NMR spectra of these compounds for the olefinic hydrogens 1" and 2" confirmed the formation of the expected $Z$ isomers.

The hydrogenation of $\mathbf{3}(\mathbf{a}, \mathbf{b}, \mathbf{c})$ yielded the compounds $4(\mathbf{a}, \mathbf{b}, \mathbf{c})$ which ${ }^{13} \mathrm{C}$ NMR spectra showed the two methylene signals at $c a . \delta 33$ and 26. The change in the multiplicity of hydrogens 2' signals from triplets to multiplets (or doublet of doublet of doublets in case of 4a, with $J_{2^{\prime}, 3^{\prime}}=10.2 \mathrm{~Hz}, J_{2^{\prime}, 1^{\prime \prime a}}=7.9$ and $J_{2^{\prime}, 1^{\prime \prime} \mathrm{b}}=2.4 \mathrm{~Hz}$ ) confirmed the hydrogenation.

The first lactone ring was closed by reaction of the esters $4(\mathbf{a}, \mathbf{b}, \mathbf{c})$ with sulphuric acid (2\%) in 1,4-dioxane under reflux (Scheme 1). The formation of compounds 5 $(\mathbf{a}, \mathbf{b}, \mathbf{c})$ was confirmed by the $\mathrm{v}_{\mathrm{O}-\mathrm{H}}\left(\mathrm{ca} .3405 \mathrm{~cm}^{-1}\right)$ and $v_{C=0}\left(c a .1781 \mathrm{~cm}^{-1}\right)$ in the infrared spectra. The several duplicated characteristic signal groups in the NMR spectra indicated the formation of mixtures of C-8 epimers. For example, two broad signals were observed for the $\mathrm{OH}$ groups (at $c a . \delta 1.7$ and 1.6 for $\mathrm{OH}$ in $\alpha$ and $\beta$, at a $1: 2$ proportion, respectively) in the ${ }^{1} \mathrm{H}$ NMR spectra. The identity of the major product was established by the comparison of the H-8 signals, which appeared as singlets at $c a . \delta 5.54$ for the epimers with the hydroxyl group in $\beta$ position, and as doublets $\left(J_{8,1} c a .4 \mathrm{~Hz}\right)$ at $\delta 5.51$ for the minor $\alpha$ isomers. The correlated C- 8 signals were observed at $\delta 101$ and at $\delta 96$ (less intense), respectively in the ${ }^{13} \mathrm{C}$ NMR spectra of the mixtures $5(\mathbf{a}, \mathbf{b}, \mathbf{c})$.

The Jones oxidation of the compounds $\mathbf{5}(\mathbf{a}, \mathbf{b}, \mathbf{c})$ yielded the bis- $\gamma$-lactones $\mathbf{6}(\mathbf{a}, \mathbf{b}, \mathbf{c})$. The simplification of the H-1 signal multiplicity in the ${ }^{1} \mathrm{H}$ NMR of these compounds (doublets at $\delta 5.01$, with $J_{1,5}=7.8 \mathrm{~Hz}$ ) and the observation of two carbonyl signals in their ${ }^{13} \mathrm{C}$ NMR spectra (C-8 at $c a . \delta 169.9$ and C-3 at $c a . \delta$ 173.6) confirmed the oxidation at $\mathrm{C}-8$.

The exocyclic methylene group was introduced into the bis- $\gamma$-lactone skeleton using the methodology described by Parker and Johnson. ${ }^{14}$ The reaction of 6 (a, b, c) with methylmethoxymagnesium carbonate (MMC, Scheme 1), followed by the addition of hydrochloric acid yielded the carboxylic acid intermediates, which were not isolated. To the crude products (yellow oils) was added a mixture of sodium acetate, acetic acid, formaldehyde, water and diethylamine yielding the avenaciolide analogs $7(\mathbf{a}, \mathbf{b}, \mathbf{c})$.

The expected change in the chemical shifts of C-4 signals from $\delta 32$ in the ${ }^{13} \mathrm{C}$ spectra of the parent bis- $\gamma$ lactones to the range of $\delta 134$ confirmed the formation of compounds $7(\mathbf{a}, \mathbf{b}, \mathbf{c})$. As in avenaciolide ${ }^{1} \mathrm{H}$ NMR spectrum,,$^{15}$ the vinylic hydrogens 1 " of the analogs 7 (a, b, c) originated two doublets at $\delta 5.87(\mathrm{H} \beta)$ and at $c a$. $\delta 6.47(\mathrm{H} \alpha)$ in their spectra. The correlated carbon signals
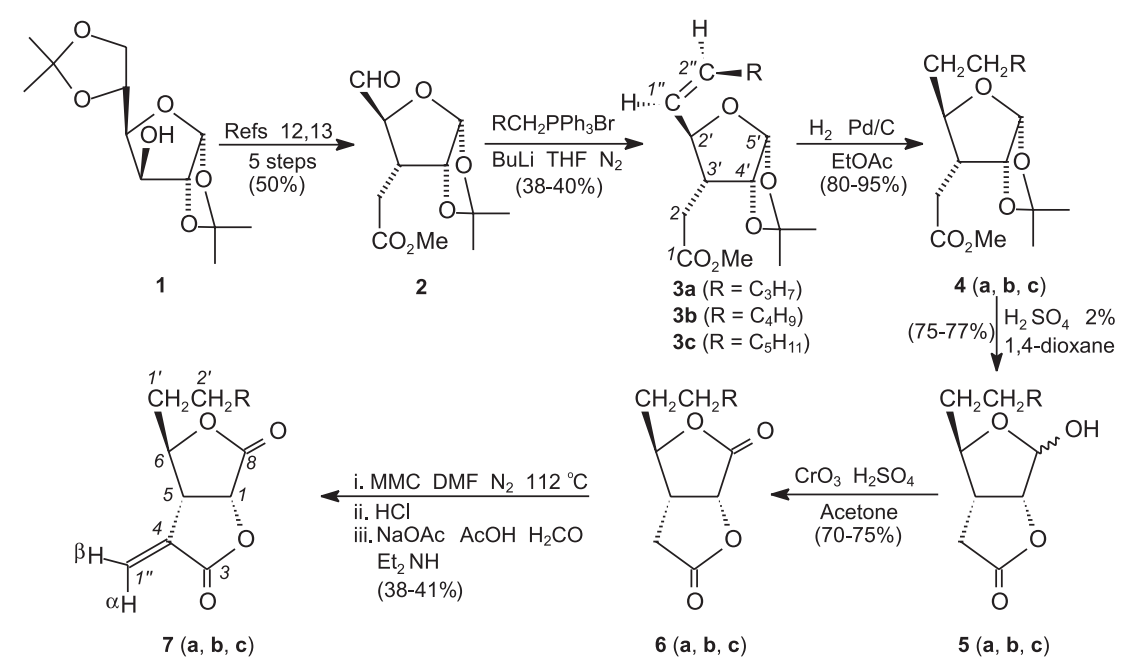

Scheme 1. 
were observed at $\delta 126.5$ in the ${ }^{13} \mathrm{C}$ NMR spectra of $7 \mathbf{a}$ and $\mathbf{7 c}$, and at $\delta 124.0$ in the spectrum of $\mathbf{7 b}$.

The activity of compounds $\mathbf{3}$ to $\mathbf{7}$ on the germination of conidia of Colletotrichum gloesporioides was evaluated in vitro. In Brazil, this is one of the most important pathogens causing great losses in fruit production. The fungicide Benlate, which active principle (benomyl) was used as a positive check treatment in the biological tests here described, was widely used in Brazil for the control of several plant diseases caused by various fungal species but is no longer in the market. Our interests in alternatives for the control of Colletotrichum species has increased with the resistance presented by these fungi to most commercially available agrochemicals. ${ }^{16}$

Under the test conditions, compounds 3 to $\mathbf{6}(\mathbf{a}, \mathbf{b}, \mathbf{c})$ were inactive, while the bis- $\gamma$-lactones $7(\mathbf{a}, \mathbf{b}, \mathbf{c})$ showed inhibitions of 39,40 and $46 \%$, respectively, with respect to benomyl. It is important to point out that the compounds tested have little solubility in the aqueous medium, which interferes in their dispersion on the Petri dishes. Nevertheless, these results indicate that not only the bis$\gamma$-lactone skeleton is important for the antifungal activity of these compounds but also it depends on the presence of the exocyclic double bond, probably due to a Michael type addition reaction with the fungi enzymes. It is noteworthy that avenaciolide inhibits the glutamate transport in mitochondria by irreversibly bonding to the - $\mathrm{SH}$ groups of the glutamate carrier located in a hydrophobic part of the membrane. ${ }^{2}$

As expected, the length of the alkyl side chain interferes in the magnitude of the biological activities presented by these compounds, confirming the suggestion made by Meyer and Vignais ${ }^{2}$ that the longer side chain of avenaciolide (compared to the inactive ethisolide) should increase the interactions with the lipophilic part of the membrane, exposing the methylene group to the reaction with the thiol groups of the glutamate carrier.

\section{Experimental}

\section{General}

Solvents were distilled before use and dried according to standard procedures. Reactions were monitored by TLC on precoated plates with silica gel $60 \mathrm{G}$ (Sigma - Aldrich). Column chromatography was carried out on silica gel 60 (70-230 mesh). Microanalyses were performed in a PERKIN ELMER 2400 elemental analyzer. IR spectra were recorded on a PERKIN ELMER FT-IR 1000 spectrometer, scanning from 625 to $4000 \mathrm{~cm}^{-1}$, in $\mathrm{KBr}$ disks or, in the case of oily compounds, as thin films on
$\mathrm{NaCl}$ plates. The NMR spectra were recorded on a Bruker Avance DRX 400 or a Bruker Avance 200 or a VARIAN $300 \mathrm{MHz}$, using $\mathrm{CDCl}_{3}$ as solvent and TMS as the internal standard. Chemical shifts $(\delta)$ are given in ppm and coupling constants $(J)$ values are in Hertz $(\mathrm{Hz})$.

\section{Synthesis of compounds}

The aldehyde 2 was prepared from diacetone-Dglucose (1) as described in the literature..$^{12,13}$

General procedures for the synthesis of olefins $3(\boldsymbol{a}, \boldsymbol{b}, \boldsymbol{c})$

The alkyltriphenylphosphonium bromides were prepared by refluxing a solution of triphenylphosphine and the alkyl bromides (1:1) in toluene, under nitrogen atmosphere for 10 hours. The solids were separated by filtration, washed with dry diethyl ether and dried under reduced pressure (70 to $80 \%$ yields). The phosphonium salts thus obtained $(0.018 \mathrm{~mol})$ were dissolved in dry THF (37 $\mathrm{mL})$, butyl lithium $(0.011 \mathrm{~mol})$ was added and the reaction mixtures were stirred for $0.5 \mathrm{~h}$ under a nitrogen atmosphere. A solution of the aldehyde $2(0.009 \mathrm{~mol})$ in dry THF (5.0 $\mathrm{mL}$ ) was added drop wise and the stirring continued for $3 \mathrm{~h}$ at room temperature. Elimination of the THF, addition of water and extraction with $\mathrm{Et}_{2} \mathrm{O}$ gave the crude reaction product. Purification (removal of phosphorus containing residue) was effected by chromatography on a silica gel column (hexane: EtOAc 3:1).

Data for methyl (2'R,3'R,4'R,5'R)-2-[(Z)-2'-penten-1'”$y l-4$ ',5'-isopropilidenedeoxy-tetrahydrofuran-3'-yl] acetate, $3 a$

White solid, $38 \%$ yield; mp $59.4-61.4{ }^{\circ} \mathrm{C} ; R_{\mathrm{f}} 0.64$ (hexane:EtOAc 3:1); IR (KBr) $v_{\max } / \mathrm{cm}^{-1}: 3014,1735$, 1437, 1373, 1332, 1170, 872; ${ }^{1} \mathrm{H}$ NMR (200 MHz, $\left.\mathrm{CDCl}_{3}\right)$ $\delta 5.84\left(\mathrm{~d},{ }^{3} J_{H 5}, H 4,4.1,1 \mathrm{H}, \mathrm{H} 5^{\prime}\right), 5.69\left(\mathrm{dt},{ }^{3} J_{H 2}{ }^{\prime \prime}-H_{1}, 10.9\right.$,

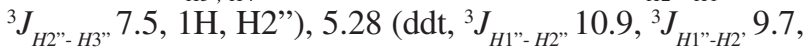
$\left.{ }^{4} J_{H 1}{ }^{\prime \prime-H 3}, 1.5,1 \mathrm{H}, \mathrm{H} 1^{\prime \prime}\right), 4.77\left(\mathrm{t},{ }^{3} J_{H 4^{\prime}-H 3^{\prime}}={ }^{3} J_{H 4^{\prime}-H 5^{\prime}}, 4.1,1 \mathrm{H}\right.$, H4'), 4.53 (t, $\left.{ }^{3} J_{H 2^{\prime}-H 1^{\prime}}={ }^{3} J_{H 2^{\prime}-H 3}, 9.7,1 \mathrm{H}, \mathrm{H} 2^{\prime}\right), 3.69$ (s, 3H, $\left.\mathrm{OCH}_{3}\right), 2.63\left(\mathrm{dd},{ }^{2} J_{H 2 \mathrm{a}-H 2 \mathrm{~b}} 16.7,{ }^{3} J_{H 2 \mathrm{a}-H 3}, 10.5,1 \mathrm{H}, \mathrm{H} 2 \mathrm{a}\right)$, $2.27\left(\mathrm{dd},{ }^{2} J_{H 2 \mathrm{~b}-H 2 \mathrm{a}} 16.7,{ }^{3} J_{H 2 \mathrm{~b}-H 3}, 4.1,1 \mathrm{H}, \mathrm{H} 2 \mathrm{~b}\right), 2.22-1.98$ (m, 3H, H3' and H3"), 1.53 (s, 3H, C( $\left.\left.\mathrm{CH}_{3}\right)_{2}\right), 1.50-1.39$ $(\mathrm{m}, 2 \mathrm{H}, \mathrm{H} 4 "), 1.33$ (s, 3H, $\left.\mathrm{C}\left(\mathrm{CH}_{3}\right)_{2}\right), 0.92$ (t, $\left.{ }^{3} J_{\mathrm{CH} 3-H 4}, 7.3,3 \mathrm{H}, \mathrm{CH}_{3}\right) ;{ }^{13} \mathrm{C} \mathrm{NMR}\left(50 \mathrm{MHz}, \mathrm{CDCl}_{3}\right) \delta$ 172.54 (C=O), 136.41 (C1"), 126.45 (C2”), 111.44 $\left(\underline{\mathrm{C}}\left(\mathrm{CH}_{3}\right)_{2}\right), 104.97$ (C5'), 80.57 (C4'), 75.77 (C2'), 51.75 $\left(\mathrm{OCH}_{3}\right), 46.02$ (C3'), 29.94 (C3”), 28.90 (C2), 26.62 $\left(\mathrm{C}\left(\mathrm{CH}_{3}\right)_{2}\right), 26.28\left(\mathrm{C}\left(\mathrm{CH}_{3}\right)_{2}\right), 22.10(\mathrm{C} 4 "), 13.79\left(\mathrm{CH}_{3}\right)$; Anal. Calc. for $\mathrm{C}_{15} \mathrm{H}_{24} \mathrm{O}_{5}: \mathrm{C}, 63.36 ; \mathrm{H}, 8.51 \%$. Found: $\mathrm{C}$, $63.11 ; \mathrm{H}, 8.58 \%$. 
Data for methyl (2'R,3'R,4'R, 5'R)-2-[(Z)-2'-hexen-1 ". $y l-4$ ',5'-isopropilidenedeoxy-tetrahydrofuran-3'-yl] acetate, $\mathbf{3 b}$

White solid, 38\% yield; mp 59.7-61.7 ${ }^{\circ} \mathrm{C} ; R_{\mathrm{f}} 0.64$ (hexane: EtOAc 3:1); IR ( NaCl) $v_{\text {max }} / \mathrm{cm}^{-1}: 1738,1690$, 1650, 1437, 1373, 1328, 1168, 872; ${ }^{1} \mathrm{H}$ NMR (300 MHz, $\left.\mathrm{CDCl}_{3}\right) \delta 5.84\left(\mathrm{~d},{ }^{3} J_{H 5^{\prime}-H 4}, 4.2,1 \mathrm{H}, \mathrm{H} 5^{\prime}\right), 5.68(\mathrm{dt}$, ${ }^{3} J_{H 2 "-H 1}, 11.0,{ }^{3} J_{H 2}$ "- $H 3,7.2,1 \mathrm{H}, \mathrm{H} 2$ "), 5.27 (ddt, ${ }^{3} J_{H 1}$ "- $H 2,11.0$, $\left.{ }^{3} J_{H 1}{ }^{\prime}-H 2,9.7,{ }^{4} J_{H 1},-H 3,1.5,1 \mathrm{H}, \mathrm{H} 1 "\right), 4.78\left(\mathrm{t},{ }^{3} J_{H 4^{\prime}-H 3^{\prime}}=\right.$ $\left.{ }^{3} J_{H 4^{\prime}-H 5^{\prime}}, 4.2,1 \mathrm{H}, \mathrm{H} 4^{\prime}\right), 4.52\left(\mathrm{t},{ }^{3} J_{H 2^{\prime}-H 1^{\prime}}={ }^{3} J_{H 2^{\prime}-H 3^{\prime}}, 9.7,1 \mathrm{H}\right.$, $\mathrm{H} 2$ ') 3.69 ( s, 3H, $\left.\mathrm{OCH}_{3}\right), 2.63\left(\mathrm{dd},{ }^{2} J_{H 2 \mathrm{a}-H 2 \mathrm{~b}} 16.7\right.$, $\left.{ }^{3} J_{H 2 \mathrm{a}-H 3}, 10.7,1 \mathrm{H}, \mathrm{H} 2 \mathrm{a}\right), 2.26\left(\mathrm{dd},{ }^{2} J_{H 2 \mathrm{~b}-H 2 \mathrm{a}} 16.7,{ }^{3} J_{H 2 \mathrm{~b}-H 3}\right.$, 4.2, 1H, H2b), 2.18-2.00 (m, 3H, H3' and H3"'), 1.52 (s, $\left.3 \mathrm{H}, \mathrm{C}\left(\mathrm{CH}_{3}\right)_{2}\right), 1.40-1.26$ (m, 4H, H4" and H5"), 1.32 (s, $\left.3 \mathrm{H}, \mathrm{C}\left(\mathrm{CH}_{3}\right)_{2}\right), 0.89$ (t, $\left.{ }^{3} \mathrm{~J}_{\mathrm{CH} 3-H 5}, 7.2,3 \mathrm{H}, \mathrm{CH}_{3}\right) ;{ }^{13} \mathrm{C} \mathrm{NMR}$ $\left(75 \mathrm{MHz}, \mathrm{CDCl}_{3}\right) \delta 172.67(\mathrm{C}=\mathrm{O}), 136.88\left(\mathrm{Cl}^{\prime \prime}\right), 126.46$ (C2”), $111.66\left(\underline{\mathrm{C}}\left(\mathrm{CH}_{3}\right)_{2}\right), 105.18$ (C5'), 80.78 (C4'), 75.96 (C2'), $51.98\left(\mathrm{OCH}_{3}\right), 46.23$ (C3'), 31.98 (C4”) 29.12 (C2), 27.82 (C3”), $26.83\left(\mathrm{C}\left(\mathrm{CH}_{3}\right)_{2}\right), 26.51\left(\mathrm{C}\left(\mathrm{CH}_{3}\right)_{2}\right), 22.49$ (C5"), $14.12\left(\mathrm{CH}_{3}\right)$; Anal. Calc. for $\mathrm{C}_{16} \mathrm{H}_{26} \mathrm{O}_{5}: \mathrm{C}, 64.41$; H, $8.78 \%$. Found: C, 64.93; H, 8.75\%.

Data for methyl (2'R,3'R,4'R,5'R)-2-[(Z)-2'-hepten-1 '-yl4',5'-isopropilidenedeoxy-tetrahydrofuran-3'-yl] acetate, $3 c$

White solid, $40 \%$ yield; $\mathrm{mp} 55.5-57.5^{\circ} \mathrm{C} ; R_{\mathrm{f}} 0.65$ (hexane: EtOAc 3:1); IR (KBr) $v_{\max } / \mathrm{cm}^{-1}: 3006,1730$, 1690, 1434, 1373, 1331, 1163, 881; ${ }^{1} \mathrm{H}$ NMR (400 MHz, $\left.\mathrm{CDCl}_{3}\right) \delta 5.85\left(\mathrm{~d},{ }^{3} J_{H 5^{\prime}-H_{4}}, 4.2,1 \mathrm{H}, \mathrm{H} 5^{\prime}\right), 5.69$ (dt, ${ }^{3} J_{H 2^{\prime \prime}-H 1}$ " $10.9,{ }^{3} J_{H 2 "-H 3}, 7.5,1 \mathrm{H}, \mathrm{H} 2$ "), 5.27 (ddt, ${ }^{3} J_{H 1 \text { "- } H 2}, 10.9$, $\left.{ }^{3} J_{H 1^{\prime}-H 2}, 9.7,{ }^{4} J_{H 1^{\prime}-H 3^{\prime}} 1.6,1 \mathrm{H}, \mathrm{H} 1^{\prime \prime}\right), 4.78\left(\mathrm{t},{ }^{3} J_{H 4^{\prime}-H 3^{\prime}}={ }^{3} J_{H 4^{\prime}-H 5^{\prime}}\right.$ $4.2,1 \mathrm{H}, \mathrm{H} 4^{\prime}$ ), 4.53 (t, ${ }^{3} J_{H 2^{\prime}-H 1^{\prime}}={ }^{3} J_{H 2^{\prime}-H}, 9.7,1 \mathrm{H}, \mathrm{H} 2^{\prime}$ ), 3.69 (s, 3H, $\left.\mathrm{OCH}_{3}\right), 2.62\left(\mathrm{dd},{ }^{2} J_{H 2 \mathrm{a}-H 2 \mathrm{~b}} 16.9,{ }^{3} \mathrm{~J}_{H 2 \mathrm{a}-H 3}\right.$, $10.6,1 \mathrm{H}, \mathrm{H} 2 \mathrm{a}), 2.28\left(\mathrm{dd},{ }^{2} J_{H 2 \mathrm{~b}-H 2 \mathrm{a}} 16.9,{ }^{3} J_{H 2 \mathrm{~b}-H 3}, 4.1,1 \mathrm{H}\right.$, H2b), 2.18-1.99 (m, 3H, H3' and H3"), 1.53 (s, 3H, $\left.\mathrm{C}\left(\mathrm{CH}_{3}\right)_{2}\right), 1.41-1.25$ (m, 6H, H4", H5" and H6"), 1.33 (m, $\left.3 \mathrm{H}, \mathrm{C}\left(\mathrm{CH}_{3}\right)_{2}\right), 0.89\left(\mathrm{t}, \mathrm{J}_{\mathrm{CH} 3-\mathrm{H} 6}, 7.2,3 \mathrm{H}, \mathrm{CH}_{3}\right) ;{ }^{13} \mathrm{C} \mathrm{NMR}$ (100 MHz, $\left.\mathrm{CDCl}_{3}\right) \delta 172.53(\mathrm{C}=\mathrm{O}), 136.69$ (C1"), 126.25 (C2”), $111.44\left(\underline{\mathrm{C}}\left(\mathrm{CH}_{3}\right)_{2}\right), 104.97$ (C5'), 80.58 (C4'), 75.77 (C2'), $51.74\left(\mathrm{OCH}_{3}\right), 46.03$ (C3'), 31.41 (C5”), 29.30 (C4”), 28.92 (C2), 27.88 (C3”), $26.61\left(\mathrm{C}\left(\mathrm{CH}_{3}\right)_{2}\right), 26.30$ $\left(\mathrm{C}\left(\mathrm{CH}_{3}\right)_{2}\right), 22.47$ (C6"), $13.99\left(\mathrm{CH}_{3}\right)$; Anal. Calc. for $\mathrm{C}_{17} \mathrm{H}_{28} \mathrm{O}_{5}: \mathrm{C}, 65.36 ; \mathrm{H}, 9.03 \%$. Found: C, 65.81; H, 9.12\%.

General procedures for hydrogenation of the olefinic substances to give compounds $\mathbf{4}(\boldsymbol{a}, \boldsymbol{b}, \boldsymbol{c})$

To $0.035 \mathrm{~mol} \mathrm{~L}^{-1}$ solutions of the olefins $\mathbf{3}(\mathbf{a}, \mathbf{b}, \mathbf{c})$ in ethyl acetate $(100 \mathrm{~mL})$ were added $50 \mathrm{mg}$ of $\mathrm{Pd} / \mathrm{C}(10 \%)$. The suspensions were stirred for $16 \mathrm{~h}$ at room temperature under hydrogen atmosphere. The mixtures were filtered and the filtrates were evaporated.
Data for methyl (2'R, 3'R,4'R, 5'R)-2-[2'-pentyl-4',5'isopropilidenedeoxytetrahydrofuran-3'-yl] acetate, $4 a$

Colorless oil, 80\% yield; $R_{\mathrm{f}} 0.64$ (hexane: EtOAc 3:1); IR $(\mathrm{NaCl}) v_{\max } / \mathrm{cm}^{-1}: 1739,1437,1373,1168,1021,874$; ${ }^{1} \mathrm{H}$ NMR (400 MHz, $\left.\mathrm{CDCl}_{3}\right) \delta 5.80\left(\mathrm{~d},{ }^{3} J_{H 5^{\prime}-\mathrm{H}}, 3.8,1 \mathrm{H}\right.$, H5'), 4.75 (dd, ${ }^{3} J_{H 4^{\prime}-H 3^{\prime}}, 4.5,{ }^{3} J_{H 4^{\prime}-H 5}, 3.8,1 \mathrm{H}, \mathrm{H} 4^{\prime}$ ), 3.75 (ddd,

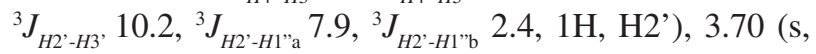
$\left.3 \mathrm{H}, \mathrm{OCH}_{3}\right) 2.66\left(\mathrm{dd},{ }^{2} J_{H 2 \mathrm{a}-H 2 \mathrm{~b}} 16.9,{ }^{3} J_{H 2 \mathrm{a}-H 3}, 10.2,1 \mathrm{H}, \mathrm{H} 2 \mathrm{a}\right)$, $2.32\left(\mathrm{dd},{ }^{2} J_{H 2 \mathrm{~b}-H 2 \mathrm{a}} 16.9,{ }^{3} J_{H 2 \mathrm{~b}-H 3}, 4.5,1 \mathrm{H}, \mathrm{H} 2 \mathrm{~b}\right), 2.04$ (tt, $\left.{ }^{3} J_{H 3^{\prime}-H 2^{\prime}}={ }^{3} J_{H 3^{\prime}-H 2 \mathrm{a}} 10.2,{ }^{3} J_{H 3^{\prime}-H 4^{\prime}}={ }^{3} J_{H 3^{\prime}-H 2 \mathrm{~b}} 4.5,1 \mathrm{H}, \mathrm{H} 3{ }^{\prime}\right), 1.58-$ 1.49 (m, 2H, H1"), 1.48 (s, 3H, C $\left.\left(\mathrm{CH}_{3}\right)_{2}\right), 1.46-1.33$ (m, 2H, H2"), 1.31 (s, 3H, C( $\left.\left.\mathrm{CH}_{3}\right)_{2}\right)$, 1.34-1.25 (m, 4H, H3" and H4"), 0.88 (t, $\left.{ }^{3} \mathrm{~J}_{\mathrm{CH} 3-\mathrm{H} 4}, 5.9,3 \mathrm{H}, \mathrm{CH}_{3}\right) ;{ }^{13} \mathrm{C}$ NMR (100 $\left.\mathrm{MHz}, \mathrm{CDCl}_{3}\right) \delta 172.70(\mathrm{C}=\mathrm{O}), 111.22\left(\underline{\mathrm{C}}\left(\mathrm{CH}_{3}\right)_{2}\right), 105.05$ (C5'), 81.45 (C4'), 80.75 (C2'), $52.15\left(\mathrm{OCH}_{3}\right), 45.22$ (C3'), 32.96 (C1”), 32.34 (C3”), 29.96 (C2), 26.93

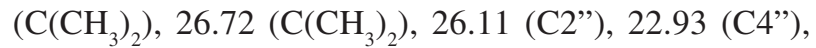
$14.40\left(\mathrm{CH}_{3}\right)$; Anal. Calc. for $\mathrm{C}_{15} \mathrm{H}_{26} \mathrm{O}_{5}: \mathrm{C}, 62.91 ; \mathrm{H}, 9.15 \%$. Found: C, 62.85; H, 9.17\%.

Data for methyl (2'R,3'R,4'R,5'R)-2-[2'-hexyl-4',5'isopropilidenedeoxytetrahydrofuran-3'-yl] acetate, $4 b$

Colorless oil, 87\% yield; $R_{\mathrm{f}} 0.63$ (hexane: EtOAc 3:1); IR $(\mathrm{NaCl}) v_{\max } / \mathrm{cm}^{-1}: 1738,1438,1372,1168,1019,874$; ${ }^{1} \mathrm{H} \mathrm{NMR}\left(300 \mathrm{MHz}, \mathrm{CDCl}_{3}\right) \delta 5.80\left(\mathrm{~d},{ }^{3} \mathrm{~J}_{\mathrm{H} 5^{\prime}-\mathrm{H}}, 3.9,1 \mathrm{H}, \mathrm{H} 5\right.$ '), $4.74\left(\mathrm{t},{ }^{3} J_{H 4^{\prime}-H 3^{\prime}}={ }^{3} J_{H 4^{\prime}-H 5^{\prime}}, 3.9,1 \mathrm{H}, \mathrm{H} 4^{\prime}\right), 3.80-3.69(\mathrm{~m}, 1 \mathrm{H}$, $\mathrm{H} 2$ ') $3.70\left(\mathrm{~s}, 3 \mathrm{H}, \mathrm{OCH}_{3}\right), 2.65\left(\mathrm{dd},{ }^{2} J_{H 2 \mathrm{a}-H 2 \mathrm{~b}} 17.0,{ }^{3} J_{H 2 \mathrm{a}-H 3}\right.$, 10.9, 1H, H2a), 2.31 (dd, ${ }^{2} J_{H 2 \mathrm{~b}-H 2 \mathrm{a}} 17.0,{ }^{3} J_{H 2 \mathrm{~b}-H 3}, 4.2,1 \mathrm{H}$, H2b), 2.08-1.99 (m, 1H, H3'), 1.60-1.45 (m, 2H, H1'), 1.44 (s, 3H, $\left.\mathrm{C}\left(\mathrm{CH}_{3}\right)_{2}\right), 1.30$ (s, 3H, $\left.\mathrm{C}\left(\mathrm{CH}_{3}\right)_{2}\right), 1.40-1.23$ (m, 8H, H2", H3", H4" and H5"), 0.86 (t, ${ }^{3} \mathrm{~J}_{\mathrm{CH} 3-H 5}$, 6.6, 3H, $\left.\mathrm{CH}_{3}\right)$; ${ }^{13} \mathrm{C}$ NMR $\left(75 \mathrm{MHz}, \mathrm{CDCl}_{3}\right) \delta 173.93(\mathrm{C}=\mathrm{O}), 111.43$ $\left(\mathrm{C}\left(\mathrm{CH}_{3}\right)_{2}\right), 104.86\left(\mathrm{C}^{\prime}\right.$ '), 81.25 (C4'), 80.55 (C2'), 52.01 $\left(\mathrm{OCH}_{3}\right), 45.02$ (C3'), 32.80 (C1'), 31.93 (C4”), 29.76 (C2),

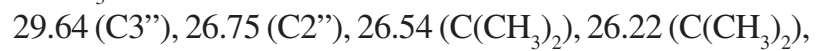
22.80 (C5"), $14.29\left(\mathrm{CH}_{3}\right)$; Anal. Calc. for $\mathrm{C}_{16} \mathrm{H}_{28} \mathrm{O}_{5}: \mathrm{C}$, 63.97; H, 9.39\%. Found: C, 63.65; H, 9.42\%.

Data for methyl (2'R,3'R,4'R,5'R)-2-[2'-heptyl-4',5'isopropilidenedeoxytetrahydrofuran-3'-yl] acetate, $4 c$

Colorless oil, $95 \%$ yield; $R_{\mathrm{f}} 0.64$ (hexane: EtOAc 3:1); IR $(\mathrm{NaCl}) v_{\max } / \mathrm{cm}^{-1}: 1739,1436,1372,1167,1020,875$; ${ }^{1} \mathrm{H}$ NMR $\left(200 \mathrm{MHz} \mathrm{CDCl}_{3}\right) \delta 5.80\left(\mathrm{~d},{ }^{3} \mathrm{~J}_{H 5^{5},{ }_{4},}, 3.9,1 \mathrm{H}\right.$, H5'), 4.74 (t, $\left.{ }^{3} J_{H 4^{\prime}-H 3^{\prime}}={ }^{3} J_{H 4^{\prime}-H 5^{\prime}}, 4.3,1 \mathrm{H}, \mathrm{H} 4^{\prime}\right), 3.80-3.70$ (m, 1H, H2') $3.71\left(\mathrm{~s}, 3 \mathrm{H}, \mathrm{OCH}_{3}\right), 2.66\left(\mathrm{dd},{ }^{2} J_{H 2 \mathrm{a}-H 2 \mathrm{~b}} 16.8\right.$, $\left.{ }^{3} J_{H 2 \mathrm{a}-H 3}, 10.2,1 \mathrm{H}, \mathrm{H} 2 \mathrm{a}\right), 2.31\left(\mathrm{dd},{ }^{2} J_{H 2 \mathrm{~b}-H 2 \mathrm{a}} 16.8,{ }^{3} J_{H 2 \mathrm{~b}-H 3}, 4.2\right.$, 1H, H2b), 2.11-1.97 (m, 1H, H3'), 1.56-1.50 (m, 2H, H1"), 1.50 (s, 3H, C( $\left.\left.\mathrm{CH}_{3}\right)_{2}\right), 1.31$ (s, 3H, C( $\left.\left.\mathrm{CH}_{3}\right)_{2}\right), 1.40-$ 1.24 (m, 10H, H2", H3", H4", H5" and H6"), 0.87 (t, $\left.{ }^{3} J_{\mathrm{CH} 3-\mathrm{H} 6}, 6.4,3 \mathrm{H}, \mathrm{CH}_{3}\right) ;{ }^{13} \mathrm{C} \mathrm{NMR}\left(50 \mathrm{MHz} \mathrm{CDCl}_{3}\right)$ $\delta 172.69(\mathrm{C}=\mathrm{O}), 111.22\left(\underline{\mathrm{C}}\left(\mathrm{CH}_{3}\right)_{2}\right), 104.69\left(\mathrm{C}^{\prime}\right.$ '), 81.11 
(C4'), 80.39 (C2'), $51.75\left(\mathrm{OCH}_{3}\right), 44.85$ (C3'), 32.62 (C1”), 31.79 (C5”), 29.73 (C2), 29.61 (C3”), 29.18 (C4”),

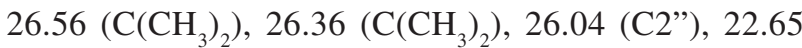
(C6"), $14.07\left(\mathrm{CH}_{3}\right)$; Anal. Calc. for $\mathrm{C}_{17} \mathrm{H}_{30} \mathrm{O}_{5}: \mathrm{C}, 64.94$; H, 9.62\%. Found: C, 64.80; H, 9.70\%.

General procedures for the synthesis of the biciclic compounds $5(\boldsymbol{a}, \boldsymbol{b}, \boldsymbol{c})$

To 0.061 mol. $\mathrm{L}^{-1}$ solutions of $\mathbf{4}(\mathbf{a}, \mathbf{b}, \mathbf{c})$ in $p$-dioxane $(40 \mathrm{~mL})$ were added $16 \mathrm{~mL}$ of $2 \%$ aqueous sulfuric acid. The mixture was refluxed for $3 \mathrm{~h}$. After cooling, $250 \mathrm{~mL}$ of $\mathrm{Et}_{2} \mathrm{O}$ were added, the organic phases were washed with water, saturated $\mathrm{NaHCO}_{3}$ solution, dried over $\mathrm{MgSO}_{4}$ and concentrated under reduced pressure. The crude products were purified by colum chromatography on silica gel (Hexane: EtOAc 1:1) to yield compounds $5(\mathbf{a}, \mathbf{b}, \mathbf{c})$ as mixtures of epimers $(\mathbf{5} \boldsymbol{\alpha}: \mathbf{5} \boldsymbol{\beta}, 1: 2)$.

Data for $(1 R, 5 R, 6 R, 8 S)$-8-hydroxy-6-pentyl-2,7-dioxabicyclo[3.3.0]octan-3-one, $\mathbf{5 a \boldsymbol { \alpha }}$ and $(1 R, 5 R, 6 R, 8 R)-8$ hydroxy-6-pentyl-2,7-dioxabicyclo[3.3.0]octan-3-one, $5 \boldsymbol{a} \boldsymbol{\beta}$

Colorless oil, 75\% yield; $R_{\mathrm{f}} 0.27$ (hexane: EtOAc 3:1); IR $(\mathrm{NaCl}) \mathrm{V}_{\max } / \mathrm{cm}^{-1}: 3401,1781,1163,1047 ;{ }^{1} \mathrm{H}$ NMR (400 $\left.\mathrm{MHz}, \mathrm{CDCl}_{3}\right) \delta 5.53(\mathrm{~s}, 1 \mathrm{H}, \mathrm{H} 8), 5.51\left(\mathrm{~d},{ }^{3} J_{H 8^{*}-H^{*}} 4.2,0.5 \mathrm{H}\right.$, H8*), 4.91-4.88 (m, 0.5H, H1*), $4.86\left(\mathrm{~d},{ }^{3} J_{H 1-H 5} 6.0,1 \mathrm{H}\right.$, $\mathrm{H} 1), 4.01-3.88$ (m, $1.5 \mathrm{H}, \mathrm{H} 6$ and $\mathrm{H} 6 *), 2.91-2.58(\mathrm{~m}, 3 \mathrm{H}$, $\mathrm{H} 5, \mathrm{H} 5^{*}, \mathrm{H} 4 \mathrm{a}$ and $\left.\mathrm{H} 4 \mathrm{a}^{*}\right), 2.60-2.40(\mathrm{~m}, 1.5 \mathrm{H}, \mathrm{H} 4 \mathrm{~b}$ and H4b*), 1.77-1.69 (m, 0.5H, OH*), 1.65-1.55 (m, 1H, OH), 1.44-1.21 (m, 12H, H1', H1'*, H2', H2'*, H3', H3'*, H4' and $\mathrm{H} 4 *$ ), 0.86-0.91 (m, 4.5H, $\mathrm{CH}_{3}$ and $\left.\mathrm{CH}_{3}{ }^{*}\right) ;{ }^{13} \mathrm{C}$ NMR $\left(100 \mathrm{MHz}, \mathrm{CDCl}_{3}\right) \delta 176.70\left({ }^{*} \mathrm{C}=\mathrm{O}\right), 175.96(\mathrm{C}=\mathrm{O}), 101.11$ (C8), $95.84\left(\mathrm{C} 8^{*}\right), 88.61(\mathrm{C} 1), 88.28$ (C6), $83.58(\mathrm{C} 1 *)$, 82.49 (C6*), 42.84 (C5), 42.33 (C5*), 37.76 (C1'), 34.91 (C1'*), 34.21 (C3'), 33.57 (C3**), 31.89 (C4*), 31.79 (C4), 25.89 (C2'), 25.56 (C2'*), 22.76 (C4'), 22.74 (C4'*), 14.21 $\left(\mathrm{CH}_{3}\right), 14.19\left(\mathrm{CH}_{3}^{*}\right)$; Anal. Calc. for $\mathrm{C}_{11} \mathrm{H}_{18} \mathrm{O}_{4}: \mathrm{C}, 61.66$; H, 8.47\%. Found: C, 61.70; H, 8.60\%.

Data for (1R,5R,6R,8S)-8-hydroxy-6-hexyl-2,7-dioxa-

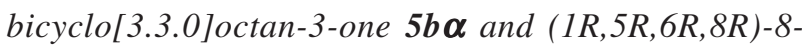
hydroxy-6-hexyl-2,7-dioxabicyclo[3.3.0]octan-3-one $\mathbf{5 b} \boldsymbol{\beta}$

Colorless oil, $75 \%$ yield; $R_{\mathrm{f}} 0.29$ (hexane: EtOAc 3:1); IR (NaCl) $v_{\max } / \mathrm{cm}^{-1}: 3404,1781,1162,1047 ;{ }^{1} \mathrm{H}$ NMR (300 $\left.\mathrm{MHz}, \mathrm{CDCl}_{3}\right) \delta 5.54(\mathrm{~s}, \quad 1 \mathrm{H}, \mathrm{H} 8), 5.51(\mathrm{~d}$, $\left.{ }^{3} J_{H 8-H 1^{*}} 3.6,0.5 \mathrm{H}, \mathrm{H} 8^{*}\right), 4.91-4.88(\mathrm{~m}, 0.5 \mathrm{H}, \mathrm{H} 1 *), 4.86(\mathrm{~d}$, $\left.{ }^{3} J_{H 1-H 5} 6.0,1 \mathrm{H}, \mathrm{H} 1\right), 4.01-3.89(\mathrm{~m}, 1.5 \mathrm{H}, \mathrm{H} 6$ and $\mathrm{H} 6 *), 2.93-$ 2.61 (m, 3H, H5, H5*, H4a and $\left.\mathrm{H}_{4} \mathrm{a}^{*}\right), 2.55-2.40(\mathrm{~m}, 1.5 \mathrm{H}$, $\mathrm{H} 4 \mathrm{~b}$ and $\left.\mathrm{H}_{4} \mathrm{~b}^{*}\right), 1.82-1.69\left(\mathrm{~m}, 0.5 \mathrm{H}, \mathrm{OH}^{*}\right), 1.68-1.52$ (m, 1H, OH), 1.42-1.20 (m, 15H, H1', H1' *, H2', H2'*, H3', $\mathrm{H} 3^{\prime} *, \mathrm{H}^{\prime}, \mathrm{H} 4{ }^{\prime} *, \mathrm{H} 5^{\prime}$ and $\left.\mathrm{H} 5^{\prime} *\right), 0.86-0.90$ (m, $4.5 \mathrm{H}, \mathrm{CH}_{3}$ and $\left.\mathrm{CH}_{3}{ }^{*}\right) ;{ }^{13} \mathrm{C} \mathrm{NMR}\left(75 \mathrm{MHz}, \mathrm{CDCl}_{3}\right) \delta 176.66\left({ }^{*} \mathrm{C}=\mathrm{O}\right)$, $175.90(\mathrm{C}=\mathrm{O}), 101.11(\mathrm{C} 8), 95.85\left(\mathrm{C} 8^{*}\right), 88.57(\mathrm{C} 1), 88.33$ (C6), 83.57 (C1*), 82.48 (C6*), 42.84 (C5), 42.36 (C5*), 37.84 (C1**), 37.86 (C1'), 34.94 (C4'), 34.21 (C4**), 33.55 (C4*), 31.93 (C4), 29.38 (C3'*), 29.28 (C3'), 26.19 (C2'), $25.86\left(\mathrm{C}^{\prime} *\right), 22.78$ (C5'), $22.79\left(\mathrm{C5}^{\prime} *\right), 14.28\left(\mathrm{CH}_{3}\right), 14.29$ $\left(\mathrm{CH}_{3}^{*}\right)$; Anal. Calc. for $\mathrm{C}_{12} \mathrm{H}_{20} \mathrm{O}_{4}: \mathrm{C}, 63.14 ; \mathrm{H}, 8.83 \%$. Found: C, 63.10; H, 9.05\%.

Data for $(1 R, 5 R, 6 R, 8 S)$-8-hydroxy-6-hepyl-2,7-dioxabicyclo[3.3.0]octan-3-one, $5 \boldsymbol{c} \boldsymbol{\alpha}$ and $(1 R, 5 R, 6 R, 8 R)-8$ hydroxy-6-heptyl-2,7-dioxabicyclo[3.3.0]octan-3-one, $5 c \beta$

Colorless oil, 77\% yield; $R_{\mathrm{F}} 0.29$ (hexane: EtOAc 3:1); IR (NaCl) $v_{\text {max }} / \mathrm{cm}^{-1}: 3408,1783,1162,1047 ;{ }^{1} \mathrm{H}$ NMR $(300$ $\left.\mathrm{MHz}, \mathrm{CDCl}_{3}\right) \quad \delta \quad 5.54(\mathrm{~s}, \quad 1 \mathrm{H}, \mathrm{H} 8), 5.51 \quad(\mathrm{~d}$, $\left.{ }^{3} J_{H 8 *-H 1^{*}} 3.9,0.5 \mathrm{H}, \mathrm{H} 8 *\right), 4.91-4.87(\mathrm{~m}, 0.5 \mathrm{H}, \mathrm{H} 1 *), 4.86(\mathrm{~d}$, $\left.{ }^{3} J_{H 1-H 5} 6.0,1 \mathrm{H}, \mathrm{H1}\right), 4.01-3.89(\mathrm{~m}, 1.5 \mathrm{H}, \mathrm{H6}$ and $\mathrm{H} 6 *), 2.94-$ 2.62 (m, 3H, H5, H5*, H4a and $\mathrm{H}_{4} \mathrm{a}^{*}$ ), 2.54-2.40 (m, 1.5H, $\mathrm{H} 4 \mathrm{~b}$ and $\left.\mathrm{H}_{4} \mathrm{~b}^{*}\right), 1.82-1.69$ (m, 0.5H, $\left.\mathrm{OH}^{*}\right), 1.66-1.54$ (m, 1H, OH), 1.40-1.23 (m, 18H, H1', H1'*, H2', H2'* H3', $\mathrm{H}^{\prime}{ }^{*}, \mathrm{H} 4{ }^{\prime}, \mathrm{H} 4{ }^{\prime}$, $, \mathrm{H} 5{ }^{\prime}, \mathrm{H} 5{ }^{\prime} *$, H6' and H6'*), 0.86-0.90 (m, 4.5H, $\mathrm{CH}_{3}$ and $\left.\left.\mathrm{CH}_{3} *\right) ;{ }^{13} \mathrm{C} \mathrm{NMR} \mathrm{(75} \mathrm{MHz,} \mathrm{CDCl}_{3}\right) \delta 176.48$ $\left({ }^{*} \mathrm{C}=\mathrm{O}\right), 175.68(\mathrm{C}=\mathrm{O}), 100.95(\mathrm{C} 8), 95.67(\mathrm{C} 8 *), 88.46(\mathrm{C} 1)$, 88.03 (C1*), 83.38 (C6), 82.30 (C6*), 42.16 (C5), 42.67 (C5*), 34.77 (C1'), 37.59 (C1'*), 33.77 (C5'), 33.79 (C5'*), 32.09 (C3'), 32.08 (C3'*), 31.77 (C4), 31.79 (C4*), 29.48 (C4**), 29.38 (C4'), 26.04 (C2**), 25.68 (C2'), 22.63 (C6'), $22.48\left(\mathrm{C}^{*} *\right), 14.07\left(\mathrm{CH}_{3}\right), 14.05\left(\mathrm{CH}_{3}^{*}\right)$; Anal. Calc. for $\mathrm{C}_{13} \mathrm{H}_{22} \mathrm{O}_{4}:$ C, 64.44; H, 9.15\%. Found: C, 64.70; H, 9.07\%. The attributions marked "**" are due to the $\alpha$ epimers. All the others refer to the major products $\beta$.

General procedures for the synthesis of bis- $\gamma$-lactones 6 $(\boldsymbol{a}, \boldsymbol{b}, \boldsymbol{c})$

The Jones reagent (prepared from $26.7 \mathrm{~g}$ of $\mathrm{CrO}_{3}$ and 23.0 $\mathrm{mL}$ of conc. $\mathrm{H}_{2} \mathrm{SO}_{4}$ and water up to $100 \mathrm{~mL}$ ) was added drop wise to $0.054 \mathrm{~mol} \mathrm{~L}^{-1}$ stirring solutions of compounds $5(\mathbf{a}, \mathbf{b}, \mathbf{c})$ in acetone $(30 \mathrm{~mL})$ till the mixtures acquired a permanent orange-brown color. Then, $50 \mathrm{~mL}$ of $\mathrm{CH}_{2} \mathrm{Cl}_{2}$ were added and stirring was continued for $10 \mathrm{~min}$, when water $(30 \mathrm{~mL})$ was added. The organic phase was washed with saturated $\mathrm{NaHCO}_{3}$ solution and with water, dried over $\mathrm{MgSO}_{4}$ and concentrated under reduced pressure to yield compounds $\mathbf{6}(\mathbf{a}, \mathbf{b}, \mathbf{c})$.

Data for (1R,5R,6R)-6-pentyl-2,7-dioxabicyclo[3.3.0] octan-3,8-dione, $\mathbf{6 a}$

White solid, $73 \%$ yield; $\mathrm{mp} 55.8-57.8{ }^{\circ} \mathrm{C} ; R_{\mathrm{f}} 0.37$ (hexane: EtOAc 3:1); IR (KBr) $v_{\max } / \mathrm{cm}^{-1}: 1795,1763$, 
1462, 1245, 1159, 1076, 999. ' $\mathrm{H}$ NMR (300 MHz, $\left.\mathrm{CDCl}_{3}\right)$; $\delta 5.01\left(\mathrm{~d},{ }^{3} J_{H 1-H 5} 7.8,1 \mathrm{H}, \mathrm{H} 1\right), 4.37-4.32(\mathrm{~m}, 1 \mathrm{H}, \mathrm{H} 6)$, 3.08-3.01 (m, 1H, H5), 2.94 (dd, ${ }^{2} J_{H 4 a-H 4 b} 17.7,{ }^{3} J_{H 4 a-H 5} 9.3$, $1 \mathrm{H}, \mathrm{H} 4 \mathrm{a}), 2.55$ (dd, $\left.{ }^{2} J_{H 4 \mathrm{~b}-H 4 \mathrm{a}} 17.7,{ }^{3} J_{H 4 \mathrm{~b}-H 5} 3.8,1 \mathrm{H}, \mathrm{H} 4 \mathrm{~b}\right)$, 1.76-1.64 (m, 2H, H1'), 1.48-1.25 (m, 6H, H2', H3' and H4'), 0.90 (t, $\left.{ }^{3} \mathrm{CH}_{\mathrm{CH}-\mathrm{H}^{\prime}} 6.7,3 \mathrm{H}, \mathrm{CH}_{3}\right) ;{ }^{13} \mathrm{C} \mathrm{NMR}(75 \mathrm{MHz}$, $\left.\mathrm{CDCl}_{3}\right) \delta 173.61(\mathrm{C}=\mathrm{O}), 169.84(\mathrm{C}=\mathrm{O}), 85.13$ (C6), 77.35 (C1), 40.64 (C5), 35.90 (C1'), 33.31 (C3'), 31.76 (C4), 25.10 (C2'), 22.91 (C4'), $14.46\left(\mathrm{CH}_{3}\right)$; Anal. Calc. for $\mathrm{C}_{11} \mathrm{H}_{16} \mathrm{O}_{4}: \mathrm{C}, 62.25 ; \mathrm{H}, 7.60 \%$. Found: C, 62.54; H, 7.99\%.

Data for $\quad(1 R, 5 R, 6 R)-6$-hexyl-2,7-dioxabicyclo [3.3.0]octan-3,8-dione, $\boldsymbol{6} \boldsymbol{b}$

White solid, $70 \%$ yield; $\mathrm{mp} 56.0-58.0^{\circ} \mathrm{C} ; R_{\mathrm{f}} 0.35$ (hexane: EtOAc 3:1); IR (NaCl) $v_{\max } / \mathrm{cm}^{-1}: 1795,1467$, 1243, 1155, 1079, 971. ${ }^{1} \mathrm{H}$ NMR $\left(300 \mathrm{MHz}, \mathrm{CDCl}_{3}\right) \delta$ $5.01\left(\mathrm{~d}, 1 \mathrm{H},{ }^{3} J_{H 1-H 5} 7.8, \mathrm{H} 1\right), 4.37-4.31(\mathrm{~m}, 1 \mathrm{H}, \mathrm{H} 6), 3.05-$ 3.02 (m, 1H, H5), 2.94 (dd, ${ }^{2} J_{H 4 a-H 4 b} 17.7,{ }^{3} J_{H 4 a-H 5} 9.4,1 \mathrm{H}$, H4a), $2.55\left(\mathrm{dd},{ }^{2} J_{H 4 \mathrm{~b}-H 4 \mathrm{a}} 17.7,{ }^{3} J_{H 4 \mathrm{~b}-H 5} 3.9,1 \mathrm{H}, \mathrm{H} 4 \mathrm{~b}\right), 1.76-$ 1.64 (m, 2H, H1'), 1.44-1.30 (m, 8H, H2', H3', H4' and H5'), 0.88 (t, $\left.{ }^{3} \mathrm{~J}_{\text {Сн- }-\mathrm{H}}, 6.93 \mathrm{H}, \mathrm{CH}_{3}\right) ;{ }^{13} \mathrm{C}$ NMR (75 MHz, $\left.\mathrm{CDCl}_{3}\right) \delta 173.67(\mathrm{C}=\mathrm{O}), 169.90(\mathrm{C}=\mathrm{O}), 85.16(\mathrm{C} 6), 77.36$ (C1), 40.63 (C5), 35.93 (C1'), 33.31 (C4'), 32.00 (C4), 29.29 (C3'), 25.38 (C2'), 22.99 (C5'), $14.56\left(\mathrm{CH}_{3}\right)$; Anal. Calc. for $\mathrm{C}_{12} \mathrm{H}_{18} \mathrm{O}_{4}$ : C, 63.70; H, 8.02\%. Found: C, 63.80; $\mathrm{H}, 7.94 \%$.

Data for (1R,5R,6R)-6-heptyl-2,7-dioxabicyclo [3.3.0]octan-3,8-dione, $6 \mathrm{c}$

White solid, $75 \%$ yield; $\mathrm{mp} 56.8-58.8{ }^{\circ} \mathrm{C} ; R_{\mathrm{f}} 0.39$ (hexane: EtOAc 3:1); IR (KBr) $v_{\max } / \mathrm{cm}^{-1}: 1783,1465$, 1246, 1144, 1081, 972; ${ }^{1} \mathrm{H}$ NMR (300 MHz, $\left.\mathrm{CDCl}_{3}\right) \delta$ $5.01\left(\mathrm{~d},{ }^{3} J_{H 1-H 5} 7.8,1 \mathrm{H}, \mathrm{H} 1\right), 4.35-4.33$ (m, 1H, H6), 3.113.00 (m, 1H, H5), 2.94 (dd, ${ }^{2} J_{H 4 \mathrm{a}-H 4 \mathrm{~b}} 17.8,{ }^{3} J_{H 4 \mathrm{a}-H 5} 9.4,1 \mathrm{H}$, H4a), $2.55\left(\mathrm{dd},{ }^{2} J_{H 4 \mathrm{~b}-H 4 \mathrm{a}} 17.8,{ }^{3} J_{H 4 \mathrm{~b}-H 5} 3.4,1 \mathrm{H}, \mathrm{H} 4 \mathrm{~b}\right), 1.76-$ 1.69 (m, 2H, H1'), 1.25-1.42 (m, 10H, H2', H3', H4', $\mathrm{H} 5$ ' and $\mathrm{H} 6$ '), 0.88 (t, $\left.{ }^{3} \mathrm{~J}_{\mathrm{CH} 3-\mathrm{H}^{6}} 6.7,3 \mathrm{H}, \mathrm{CH}_{3}\right) ;{ }^{13} \mathrm{C} \mathrm{NMR}(75$ $\left.\mathrm{MHz}, \mathrm{CDCl}_{3}\right) \delta 173.61(\mathrm{C}=\mathrm{O}), 169.83(\mathrm{C}=\mathrm{O}), 85.11(\mathrm{C} 6)$, 77.35 (C1), 40.65 (C5), 35.94 (C1'), 33.32 (C5'), 32.13 (C4), 29.60 (C3'), 29.51 (C4'), 29.30 (C2'), 23.10 (C6'), $14.61\left(\mathrm{CH}_{3}\right)$; Anal. Calc. for $\mathrm{C}_{13} \mathrm{H}_{20} \mathrm{O}_{4}: \mathrm{C}, 64.98 ; \mathrm{H}, 8.39 \%$. Found: C, 65.28; H, 8.53\%.

General procedures for the synthesis of the avenaciolide analogues $7(\boldsymbol{a}, \boldsymbol{b}, \boldsymbol{c})$

The bis-lactones $\mathbf{6}(\mathbf{a}, \mathbf{b}, \mathbf{c})(0.94 \mathrm{mmol})$ were dissolved in a $2.0 \mathrm{~mol} \mathrm{~L}^{-1}$ solution of methyl methoxymagnesium carbonate in DMF $(4.0 \mathrm{~mL})$, under $\mathrm{N}_{2}$ atmosphere. After 5 $\mathrm{h}$ under reflux $\left(120^{\circ} \mathrm{C}\right)$, the mixtures were poured into ice cold 6 mol. $\mathrm{L}^{-1} \mathrm{HCl}$ and $\mathrm{Et}_{2} \mathrm{O}(1: 5,11 \mathrm{~mL})$ and shaken until the precipitates were dissolved. The organic layers were then washed with water and dried over $\mathrm{MgSO}_{4}$. The solvent was removed under reduced pressure affording the corresponding bis- $\gamma$-lactone carboxylic acids as colorless oils. Sodium acetate $(0.2 \mathrm{~g})$ was dissolved in acetic acid $(8.0 \mathrm{~mL})$ and mixed with a solution of formalin $(6.0 \mathrm{~mL})$ and diethylamine $(2.0 \mathrm{~mL})$. A portion of this solution $(2.0$ $\mathrm{mL}$ ) was added to the bis-lactonic acids obtained and shaken vigorously until evolution of $\mathrm{CO}_{2}$ ceased (2-3 min). The mixtures were heated on a steam bath for $5 \mathrm{~min}$, cooled, and poured into water $(20 \mathrm{~mL})$ and ether $(35 \mathrm{~mL})$. The ether phases were washed with water and saturated $\mathrm{NaHCO}_{3}$ solution and dried over $\mathrm{MgSO}_{4}$. Evaporation of the ether afforded white solids, which were purified by column chromatography on silica gel with hexane/ethyl acetate 3:1, yielding compounds $\mathbf{7}(\mathbf{a}, \mathbf{b}, \mathbf{c})$.

Data for (1R,5R,6R)-6-pentyl-4-methylene-2,7-dioxabicyclo[3.3.0]octan-3,8-dione, $7 \boldsymbol{a}$

White solid, $40 \%$ yield; $\mathrm{mp} 48.2-50.2{ }^{\circ} \mathrm{C} ; R_{\mathrm{f}} 0.43$ (hexane: EtOAc 3:1); IR (NaCl) $v_{\text {max }} / \mathrm{cm}^{-1}: 1781,1664$. ${ }^{1} \mathrm{H}$ NMR $\left(300 \mathrm{MHz}, \mathrm{CDCl}_{3}\right) \delta 6.47\left(\mathrm{~d},{ }^{4} J_{H 1}{ }^{\prime \prime a-H 5} 2.4,1 \mathrm{H}\right.$,

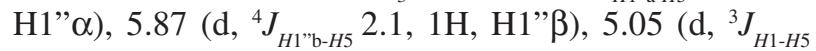
8.4, 1H, H1), 4.45-4.40 (m, 1H, H6), 3.58-3.53 (m, $1 \mathrm{H}$, H5), 1.84-1.76 (m, 2H, H1'), 1.51-1.29 (m, 6H, H2', H3' and $\mathrm{H} 4$ '), 0.91 (t, $\left.{ }^{3} \mathrm{~J}_{\mathrm{CH} 3-4^{4}} 6.8,3 \mathrm{H}, \mathrm{CH}_{3}\right) ;{ }^{13} \mathrm{C}$ NMR (75 $\left.\mathrm{MHz}, \mathrm{CDCl}_{3}\right) \delta 169.74(\mathrm{C}=\mathrm{O}), 167.49(\mathrm{C}=\mathrm{O}), 134.68$ (C4), 126.47 (C1"), 85.46 (C6), 74.61 (C1), 44.60 (C5), 36.50 (C1'), 31.73 (C3'), 25.01 (C2'), 22.93 (C4'), 14.46 $\left(\mathrm{CH}_{3}\right)$; Anal. Calc. for $\mathrm{C}_{12} \mathrm{H}_{16} \mathrm{O}_{4}: \mathrm{C}, 64.27$; $\mathrm{H}, 7.19 \%$. Found: C, 64.7; H, 7.31\%.

Data for (1R,5R,6R)-6-hexyl-4-methylene-2,7-dioxabicyclo[3.3.0]octan-3,8-dione, $7 \boldsymbol{b}$

White solid, 38\% yield; $\mathrm{mp} 50.7-52.7{ }^{\circ} \mathrm{C} ; R_{\mathrm{f}} 0.45$ (hexane: EtOAc 3:1); IR (NaCl) $v_{\max } / \mathrm{cm}^{-1}: 1781,1664$;

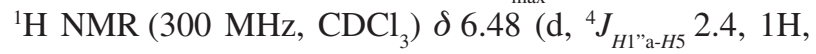

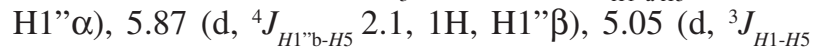
8.4, 1H, H1), 4.45-4.39 (m, 1H, H6), 3.58-3.53 (m, 1H, H5), 1.85-1.76 (m, 2H, H1'), 1.47-1.29 (m, 8H, H2', H3', $\mathrm{H} 4$ ' and $\mathrm{H} 5$ '), 0.89 (t, $\left.{ }^{3} J_{\mathrm{CH} 3-H 5^{\prime}} 6.8,3 \mathrm{H}, \mathrm{CH}_{3}\right) ;{ }^{13} \mathrm{C} \mathrm{NMR}(75$ $\left.\mathrm{MHz}, \mathrm{CDCl}_{3}\right) \delta 167.36(\mathrm{C}=\mathrm{O}), 165.08(\mathrm{C}=\mathrm{O}), 132.16$ (C4), 123.97 (C1"), 82.93 (C6), 72.04 (C1), 41.92 (C5), 33.86 (C1'), 29.35 (C4'), 26.59 (C3'), 22.62 (C2'), 20.32 (C5'), $14.89\left(\mathrm{CH}_{3}\right)$; Anal. Calc. for $\mathrm{C}_{13} \mathrm{H}_{18} \mathrm{O}_{4}: \mathrm{C}, 65.53$; H, 7.61\%. Found: C, 65.27; H, 7.73\%.

Data for (1R,5R,6R)-6-heptyl-4- methylene-2,7dioxabicyclo[3.3.0]octan-3,8-dione, $7 c$

White solid, $41 \%$ yield; $\mathrm{mp} 55.3-57.3{ }^{\circ} \mathrm{C} ; R_{\mathrm{f}} 0.44$ (hexane: EtOAc 3:1); IR (NaCl) $v_{\text {max }} / \mathrm{cm}^{-1}: 1782,1664$; 


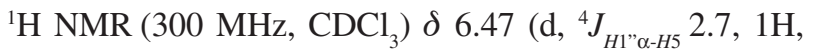

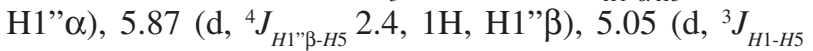
8.4, 1H, H1), 4.45-4.40 (m, 1H, H6), 3.58-3.54 (m, 1H, H5), 1.84-1.76 (m, 2H, H1'), 1.50-1.28 (m, 10H, H2', H3', H4', H5' and H6'), 0.88 (t, $\left.{ }^{3} \mathrm{~J}_{\text {СH3-H6 }} 6.8,3 \mathrm{H}, \mathrm{CH}_{3}\right) ;{ }^{13} \mathrm{C}$ $\operatorname{NMR}\left(75 \mathrm{MHz}, \mathrm{CDCl}_{3}\right) \delta 169.63(\mathrm{C}=\mathrm{O}), 167.36(\mathrm{C}=\mathrm{O})$, 134.67 (C4), 126.46 (C1”), 85.45 (C6), 74.61 (C1), 44.59 (C5), 36.54 (C1'), 32.13 (C5'), 29.56 (C3'), 29.52 (C4'), 25.33 (C2'), 23.09 (C6'), $14.61\left(\mathrm{CH}_{3}\right)$; Anal. Calc. for $\mathrm{C}_{14} \mathrm{H}_{20} \mathrm{O}_{4}: \mathrm{C}, 66.65 ; \mathrm{H}, 7.99 \%$. Found: C, 66.24; H, 8.11\%.

\section{Biological activity}

The biological activity of the bis- $\gamma$-lactones $7(\mathbf{a}, \mathbf{b}, \mathbf{c})$ and of their synthetic precursors $[3,4,5$ and $6(a, b, c)]$ were evaluated in vitro against Colletotrichum gloesporioides. The samples for test were prepared on paper disks "Blank" sterile of $6 \mathrm{~mm}$ diameter. The disks were dipped into $100 \mathrm{ppm}$ solutions of the substances in $\mathrm{CH}_{2} \mathrm{Cl}_{2}$ and the solvent was evaporated at room temperature. The same procedure was followed for the preparation of the control (benomyl). The negative check treatment was prepared with solvent only. All the samples were prepared in three repetitions. The disks were placed in the center of Petri dishes containing Colletotrichum gloesporioides conidia on potato dextrose agar (PDA) at the concentration of $4.7 \times 10^{5}$ conidia $\mathrm{mL}^{-1}$. The Petri dishes were sealed with parafilm and kept at $25^{\circ} \mathrm{C}$ under fluorescent light for 10 days. Compounds $3-6(\mathbf{a}, \mathbf{b}$, c) were inactive. After $24 \mathrm{~h}$, benomyl showed an inhibition hale of $3.90 \mathrm{~cm}$ diameter while the diameter of compounds $7(\mathbf{a}, \mathbf{b}, \mathbf{c})$ hales measured $1.51,1.56$ and $1.80 \mathrm{~cm}$, respectively. These hales were unchanged after 10 days.

\section{Conclusions}

Three new avenaciolide analogs containing side chains of different lengths and 15 new synthetic intermediates were prepared and characterized. The avenaciolide analogs were active against Colletotrichum gloesporioides, the compounds containing longer side chains being more active than the analogs with shorter side chains. The synthetic precursors, including the bicyclic compounds were inactive, confirming the importance of the bis- $\gamma$ lactone skeleton and of the conjugated exocyclic double bound for the antifungal activity.

\section{Supplementary Information}

Supplementary data are available free of charge at http://jbcs.sbq.org.br, as PDF file.

\section{Acknowledgments}

We thank the Brazilian agencies FAPEMIG for financial support, and CNPq and CAPES for students' grants.

\section{References}

1. Brookes, D.; Tidd, B. K.; Turner, W. B.; J. Chem. Soc. 1963, 68, 5385.

2. Meyer, J.; Vignais, P. M.; Biochim. Biophys. Acta 1973, 325, 375.

3. McGivan , J. D.; Chappell, J. B.; Biochem. J. 1970, 116, 37.

4. Kuo, J. F.; Dill, I. K.; Holmlund, C. E.; Bohonos, N.; Biochem. Pharmacol. 1968, 17, 345.

5. Harris, E. J.; Wimhurst, J. M.; Nature New Biology 1973, 245, 271.

6. Wimhurst, J. M.; Harris, E. J.; Biochim. Biophys. Acta 1976, 437, 51.

7. Litwinska, D.; Szczesna-Kaczmarek, A.; Popinigis, J.; Int. J. Biochem. 1984, 16, 943.

8. Aldridge, D. C.; Turner, W. B.; J. Chem. Soc. (C) 1971, 2431.

9. Martín, V. S.; Rodríguez, C. M.; Martín, T.; Org. Prep. Proced. Int. 1998, 30, 291.

10. Blot, V.; Reboul, V.; Metzner, P.; Eur. J. Org. Chem. 2006, 8, 1934; Braukmuller, S.; Bruckner, R.; Eur. J. Org. Chem. 2006, 9, 2110.

11. Anderson, R. C.; Fraser-Reid, B.; J. Am. Chem. Soc. 1975, 97 , 3870 .

12. Czernecki, S.; Georgoulouis, C.; Stevens, C. L.; Vijayakumaran, K., Tetrahedron Lett. 1985, 26, 1699.

13. Rosenthal, A.; Nguyen, L.; J. Org. Chem. 1969, 34 , 1029.

14. Parker,W. L.; Johnson, F.; J.Org. Chem. 1973, 38, 2489.

15. Brookes, D.; Sternhell, S.; Tidd, B. K.; Turner, W. B.; Aust. J. Chem. 1965, 18, 373.

16. Keinath, A. P.; Zitter, T. A.; Plant Disease 1998, 82, 479.

Received: July 25, 2006

Web Release Date: February 15, 2007 